\title{
Lo que el Aprendizaje para la Creatividad Exige a la Gestión Institucional en Centros Educativos: Aprendizajes Derivados de una Línea de Investigación ${ }^{1}$
}

\section{Demands From Required Conditions for Creative Development Over Institutional Management of Schools: Observed Characteristics That Emerge From more Than a Decade of Investigations}

\author{
Ma. Isidora Mena* y Ruby Vizcarra**
}

\begin{abstract}
Resumen
Hay contextos que facilitan la creatividad y otros que la dificultan. Pareciera que la creatividad, -potencial de todo ser humano-, es susceptible de inhibirse ante contextos que la reprimen, castigan o ignoran como posibilidad. Lo refrenda el que muchas personas no se sienten creativas, y sólo después de indagar en dimensiones privadas o cotidianas de la creatividad como la fantasía, sexualidad, relaciones humanas o cocinar, reconocen que, en efecto, crear es una actividad que también ellos realizan.

Este artículo es un intento por buscar pistas sobre contextos que permiten desarrollar la creatividad, en una serie de estudios realizados entre 1981 y 2004 en la Pontificia Universidad Católica de Chile. El contexto estudiado es el escolar: revisiones teóricas, estudios etnográficos y aplicaciones estudiadas cuasiexperimentalmente, constituyen una línea de estudio en la que cabe discutir ciertas hipótesis respecto a los contextos escolares que facilitan la actividad creadora.
\end{abstract}

Palabras claves: Creatividad, contexto, aprendizaje significativo, cultura escolar.

\footnotetext{
Abstract

Creativity appears to be a human potential, but has specific requirements for its development and open expression. In fact, there are contexts that promote creativity and others that don't; these are contexts in which this ability is repressed, punished or ignored as an educational resource. This can be appreciate when people who don't consider themselves as creative persons are asked about fantasies in private

1 Fondecyt 1010931 Santiago, Chile 2004. Colabora en este artículo Ps. Angélica Sotomayor, Psicóloga P. Universidad Católica de Chile.

* Psicóloga Escuela de Psicología, Pontificia Universidad Católica de Chile. email: imenae@uc.cl,

** Profesora Facultad de Educación, Pontificia Universidad Católica de Chile. email: rvizcarr@uc.cl
} 
domains as sexuality, cooking or other, they realize that in fact they do have expressions of creativity in these specific, more private domains.

This article intends to search clues related to contexts that facilitate creativity's open expression by a review of surveys that these authors developed, between 1981 and 2004 as investigators of the Pontificia Universidad Católica de Chile. These studies focused in school contexts. This revision includes: theoretical reviews, ethnographic studies, cuasi-experimental surveys, strategic applications in educational contexts, and, as a whole, gives place to the creation of an investigation line in which it's possible to discuss some hypothesis about observed school contexts characteristics which promotes creativity development in students.

Key words: Creativity, context, significant learning, educational institutions culture.

\section{Definición del problema}

\section{El contexto escolar como inhibidor del potencial creativo}

La escuela parece ser uno de los contextos que inhiben y bloquean la creatividad. Estudios previos la han descrito como una institución cuya organización y cultura desfavorece la creatividad (Lemaitre, Mena, Arancibia, Vásquez et al., citados en Mena, 1995). Algunos han sugerido que ya en cuarto básico la creatividad ha disminuido en comparación con la que los niños demuestran al iniciar la escuela.

Un estudio etnográfico de sala de clases (Vásquez et al., 1992, en Mena, 1992) identifica tres factores de la cultura escolar que inhiben la creatividad:

- El primero se denominó “Todo cocinado, o la comida lista y en la boca", y se refiere a que no se necesita crear para resolver los desafíos escolares.

- El segundo, “Disociación o monólogos paralelos". Plantea que en la escuela no se producen ni estimulan relaciones y que, por el contrario, se muestra una realidad parcelada en asignaturas, en unidades de materia.

- El tercer factor se tituló "El conflicto es conflictivo", muestra como el conflicto se inhibe y tiene la connotación de indeseable.
- Letelier (en López, 2001) agrega un cuarto factor: "Formalismo, o la apariencia es mejor que nada", refiriéndose al hacer rígido y formal, un modo que hace como si se hicieran actividades intelectuales y sociales que en rigor, son formas, sin desafío ni trascendencia.

El mismo López (2001) cita a Amabile y Hennessey quienes proponen cinco puntos que anulan la creatividad en la sala de clases:

- trabajar por una recompensa externa,

- promover competencia entre pares,

- destacar la evaluación,

- supervisión excesiva, y

- diseños con poca posibilidad de elección.

\section{Antecedentes Teóricos}

\section{Demandas sociales por creatividad en la escuela}

En la década de los noventa, en Chile se inicia la reforma educativa en su dimensión pedagógica, y tal como en otros países, la creatividad se transforma en un objetivo transversal de la educación. Proveniente de diversos campos sociales, a inicios de los noventa había una gran demanda de creatividad en la escuela. La tesis Doctoral de I. Mena, "Creatividad y re- 
flexión ética: una aproximación desde las demandas sociales" (1995) exploró, a través de un estudio cualitativo basado en grupos focales, los campos sociales que en 1995 demandaban creatividad al sistema educacional. Se estudiaron los campos político, económico, productivo, medio ambiente, educativo, científico y artístico. La tesis sostenía que esta demanda no era coincidente entre los diversos campos, y que lo que unos llamaban creatividad no necesariamente correspondía a los significados que otros le daban. Asimismo, se postulaba la hipótesis de que entre las distintas demandas había tensiones importantes. El estudio parecía relevante, ya que si se convenía que la escuela inhibía la creatividad, y que el contexto y la cultura escolar son los que la reprimen, hacer un cambio en esta última daría como resultado la estimulación de la creatividad, si es que había una demanda real. La tesis corroboró la hipótesis de diferentes significados para el término creatividad, pero advirtió que si bien los distintos planteamientos se tensionaban en algunos niveles, coincidían mucho en niveles más profundos. Se detectó acuerdo, por ejemplo, en que la humanidad necesita de una capacidad masiva del potencial de creatividad para sobrevivir y lograr equidad en el siglo venidero. Las condiciones productivas y socioeconómicas actuales y proyectadas así lo demandarían.

En cuanto a la gestión de contextos, del estudio de la demanda de creatividad, derivaron seis principios culturalmente pertinentes ${ }^{2}$ para la gestión en un contexto educativo que fomente la creatividad:

1. Gestionar con participación descentralizada, funcionamiento en equipos y coordinación en función de objetivos.
2. Tender a contextos flexibles.

3. Valorar la eficacia a través de evaluación e incentivos.

4. Promover identidad, diversidad y enfrentamiento del conflicto.

5. Proveer de recursos y tecnología.

6. Promover el desarrollo profesional en el tema de la enseñanza y la gestión para la creatividad.

\section{Intervenciones globales para la creatividad en contextos escolares en conflicto.}

Apoyados en la demanda de creatividad dirigida al sistema educacional, se desarrolló e implementó un proyecto en tres liceos muy conflictivos y de bajo rendimiento. El proyecto evaluó el impacto de una intervención que tenía como objetivo mejorar el contexto educativo en función de la creatividad (Fondecyt 1980610, 1999-2002). Con mediciones pre - post y con estudios cualitativos de proceso, se demostró que la estrategia tuvo bajo éxito. Además, se pudo conocer, comprender y derivar hipótesis respecto de diversos factores del contexto educativo que afectan negativamente las posibilidades de desarrollo institucional.

Buscando un marco desde el cual analizar estos contextos, se combinaron los principios derivados del estudio de demandas con las propuestas de Dyer y Mayer (2000).

Dyer y Meyer proponen que los grupos creativos tienen una relación orgánica que permite crecer, florecer y dar frutos (crear). Ellos combinan una rigurosa organización (organicidad), con gran libertad y posibilidad de equivocación.

2 Término que usa Sternberg, R. para referirse a conceptos que tienen significado para un grupo cultural dado. 
Esta "rigurosa organización" se caracteriza por tener:

- Capacidad comunicativa.

- Capacidad de congruencia y honestidad.

- Capacidad de responsabilizarse "comprendiendo el sentido", y delegar "explicando el sentido".

- Capacidad de seguir instrucciones de otros, así como de darlas.

- Procesos de evaluación.

- Costo e incentivo de respuesta.

- Exigencia.

La "gran libertad y posibilidad de equivocación" implica:

- Valoración del error como fuente de conocimiento: mecanismos para detectarlo, para incentivar su reconocimiento y para repararlo.

- Planificación de la posibilidad de hacer cambios de giro, de ítem y de tiempos (comités de evaluación de cambios durante el proceso, caja chica de tiempos, entre otros).

- Igualdad de derechos básicos (a dar ideas, a hacer críticas, a responsabilizarse, a ser honesto, a preguntar, a opinar, a saber).

- Jerarquías más horizontales, que permitan la comunicación y eviten los excesos de poder.

\section{Resultados del análisis.}

\section{a) "Cumpliendo para el patrón"}

Una de las características más llamativas que caracterizaron a las comunidades estudiadas, así como al sistema educacional y municipal que las contextualiza, es un estilo de gestión marcado por la jerarquía y la centralización, así como la nula coordinación entre las distintas acciones que se realizan. Sorprendentemente, logran funcionar en una actividad que podría ser descrita mediante una ilustrativa metáfora: el juego individual, que se despliega paralelo al de los demás, propio de la etapa en que aún no se desarrolla el juego social.

En estas comunidades, las acciones cotidianas no se realizan en función de un proyecto propio y objetivos institucionales, sino en función de disposiciones que provienen de los programas oficiales de estudio, de las normativas del sistema educacional, que a su vez, están permanentemente fiscalizadas por las peticiones de la provincia y de las normativas del municipio. Estas normativas, más que supervisar, actúan permitiendo o no las iniciativas que se le consultan.

Este accionar centralizado y jerárquico, incluye asimismo tres características que lo convierten en un factor que imposibilita la creatividad:

- es autoritario,

- la autoridad está deslegitimada, y

- los niveles superiores demandan tareas que resultan imposibles de ser realizadas, ya sea por tiempo, por disposiciones administrativas o por alguna dificultad.

Esto provoca en los distintos funcionarios la sensación de estar en permanente deuda frente a una tarea que descalifican, pero que por ser asignada por una autoridad, (que también descalifican), tiene poder sobre sus sueldos y trato profesional, y por ende, de sus vidas. El modo de accionar es del tipo "como si", en el que prevalece fuertemente una forma de actuar marcada por la superficialidad y a la que se otorga muy poco tiempo. En ningún momento se desarrollan tareas con objetivos propios, donde exista una motivación real e intrínseca. "Cumpliendo para el patrón" es el título de la novela. 
Este tipo de funcionamiento jerárquico, autoritario y sin objetivos compartidos se reproduce hacia los alumnos cuando se les asigna una función de gestión, tal como centros de alumnos, consejos de curso o la organización de un evento. Lo único que no se repite es la exigencia indebida, pues en general les proponen objetivos incluso demasiado simples. Sin embargo, en estas comunidades esto no parece constituir un problema mayor, ya que ni los alumnos ni los apoderados están, en rigor, en la cadena de gestión. Su participación es casi nula y son únicamente receptores, pasivos y espectadores ante el conocimiento que supuestamente les entrega el liceo.

\section{b) "Atenerse a la norma como modo de sobrevivencia"}

Normativas duras y rígidas logran que el sistema opere y se mantenga, pese a la gran desorganización entre las diversas acciones. Es posible sostener la hipótesis de que la representación social de quienes trabajan en estas comunidades escolares está estructurada por la existencia de normativas rígidas que se imponen con autoritarismo, inhibiendo por lo tanto, las propuestas creativas. Junto a estas normas, se advirtió que existe una gran posibilidad de trasgresión de las mismas para quienes están en cargos con poder. Esto favorece el desarrollo de un ambiente desorganizado y difícilmente predecible, que se da en paralelo al miedo y la incertidumbre laboral, producto de las decisiones fortuitas y arbitrarias que toman las autoridades. De esta manera, atenerse a la norma aparece como el único recurso conocido al cual poder "echar mano" en momentos de crisis.

\section{c) "Evaluación como herramienta de dominación"}

El sistema de evaluación tal como se propone es parte de un paradigma moderno y profesional, que se distingue profundamen- te del paradigma burocrático y jerárquico que se describía anteriormente. Sin embargo, cuando la evaluación aparece como tema para el desarrollo institucional, se genera en estos contextos gran tensión y contradicción, ya que se considera opuesta a la creatividad. Incluso, muchas veces es utilizada por las autoridades como mecanismo de control. La hipótesis que se sostiene es que el significado que se asigna a la evaluación proviene de representaciones de autoritarismo que no otorgan poder ni responsabilidad a los potenciales sujetos de la evaluación. De esta manera, estos últimos quedan como víctimas de la evaluación, la que se convierte en una herramienta más para la dominación.

\section{d) Sin metas propias, no hay creatividad.}

Los colegios deben cumplir con demandas y actividades de proyectos que pertenecen a «otros», como el ministerio, la secretaría, el municipio y los departamentos provinciales. Esto implica que los establecimientos no tienen un proyecto propio para cuyas metas quieran lograr calidad y dedicar esfuerzo; en cambio, funcionan sobre objetivos ajenos, que escasamente los representan y/o comparten. Sin esta motivación, obviamente la creatividad no se activa. Hace falta autonomía para que los colegios estén en condiciones de desarrollar coherencia en sus acciones y puedan establecer metas y estándares de calidad que otorguen una motivación institucional y pueda así activarse, llena de sentido, la creatividad.

\section{e) "Procedimientos, normas y formalidad sobre personas, diálogo y comunidad"}

El sistema burocrático con el que se organizó el sistema educacional prioriza procedimientos y formalidades en detrimento de las personas y sus particularidades. El autoritarismo, por su parte, infunde temor a expresarse y dar ideas, más aún si difieren de las del resto. Estas dos características hacen que el silencio y la homogeneidad cumplan una función protectora. 
La necesidad de identidad para el trabajo profesional se advierte, sin embargo, cuando se invita a los actores a incluir sus propios temas históricos y motivaciones para el diseño de su pedagogía. Los proyectos que se basaron en temas históricos, propios de los profesores fueron mucho mejor implementados y lograron desarrollarse a pesar de las dificultades (tutorías, paseos de estudios, aprender a través de la cocina, intercambios metodológicos).

Respecto al mismo tema, la función normalizadora y moralizadora que tiene en Chile la cultura escolar desde los inicios de la escuela como función estatal, aleja el dialogo cultural que legitima y valora las identidades, las diversidades vitales, culturales, lingüísticas y morales. En todos estos colegios se descalifica la identidad del alumnado, se habla de estar trabajando con "un material muy malo", refiriéndose a las consecuencias de la pobreza y de la situación social. Existe una descalificación que aumenta fuertemente en algunos casos cuando se sugiere hacer realidad el discurso de la co-construcción de conocimientos, del diálogo legitimante, de la responsabilización a los alumnos de actividades de curso, etc. Ahí aparece un discurso muy afectivo y violento.

\section{f) "Fobia al conflicto"}

La impresión que dejó la estadía en estas tres comunidades, es de una verdadera fobia al conflicto, que comienza por el nombre del mismo. Tensión es una palabra asociada al conflicto, que ilustra la significación que en este medio se le otorga al conflicto. Una posible línea interpretativa es que en el sistema subsiste un nivel de conflicto tan alto, que no tolera agregar nada más. Otra, es que la desesperanza funciona como defensa, lo que explicaría la superficialidad con que se analizan las tensiones. Esto protege al sistema de darse cuenta de las profundas contradicciones, vacíos organizativos y carencias en las que conviven.

\section{g) "Pobreza en herramientas de gestión"}

Mucha precariedad en recursos de gestión. Los directivos no saben de gestión, y la experiencia que tienen se remite a instituciones muy distintas a las de un liceo (escuelas rurales, inspectoría en una escuela básica, por ejemplo). La gestión de aula de los profesores, en el sentido de gestionar el conocimiento, es un tema que aun no se instala.

\section{h) "Crítica al mal funcionamiento, no al sistema de funcionamiento"}

La creatividad, si bien es muy bien recibida en un inicio, es bastante descalificada a la larga como un objetivo prioritario. La creatividad se rechaza a poco andar, como algo que viene más a complicar que a resolver el problema de cómo operar mejor en el mismo viejo paradigma nunca alcanzado. Se podría hipotetizar, en el sentido de la representación de creatividad que se tiene en general, que ésta tiene que ver con algo secundario a las metas y objetivos "más importantes" de la escuela; como algo que no se encuentra asociado a la gestión.

\section{i) "La creatividad: ¿una mala estrategia} para sistemas escolares en conflicto?"

Ninguno de los principios conocidos o asociados con contextos creativos están presentes en estas instituciones y, por el contrario, lo están sus opuestos. La creatividad no parece ser tampoco la estrategia para apoyarlos.

\section{Aprender para crear}

Entre los años cincuenta y los ochenta aproximadamente, hubo en la comunidad científica un gran esmero por el estudio de la preciada creatividad, augurando la era de la sociedad del conocimiento. El año 2000, sin embargo, creatividad ya no es la palabra clave, sino aprendizaje. Ser capaz de aprender permanentemente en un mundo cam- 
biante, se torna en un capital para las personas y para las empresas. El sistema escolar es demandado para enseñar a aprender, tanto en términos de conocimientos declarativos o contenidos básicos, que permiten entender el funcionamiento de la realidad que conocemos, como en términos de habilidades cognoscitivas, que permiten analizar, relacionar, comparar, jerarquizar, sintetizar y transferir.

Nuestra investigación más reciente (Fondecyt 1010981), enfocó la creatividad en el aprendizaje. Nos interesó explorar más profundamente cómo afecta en la adquisición de conocimiento declarativo y procedural del programa de estudios oficial, una enseñanza que apuntara a lo que llamamos "aprender para crear". La metodología para la enseñanza creativa que se propuso se probó experimentalmente en función de su impacto en los alumnos. Esta metodología propone actuar sobre las dimensiones relación pedagógica, formación en habilidades para crear y selección de contenidos.

Las dos primeras dimensiones, relación pedagógica y habilidades para crear, son aspectos conocidos en las metodologías para la creatividad, y se asocian a las condiciones de aceptación, congruencia, empatía y acoplamiento para la actualización, de las que habla Rogers (1976). Estas dimensiones se asocian asimismo al modelo de factores cognitivos y afectivos que ha desarrollado Mena y Antonijevic (1990, 1994, 1995). Respecto a los contenidos, proponen que la posibilidad de crear a partir de un determinado saber estaría dada por la profundidad con que se maneja el nuevo saber. Esta profundidad estaría determinada, a su vez, por la comprensión de las tensiones y relaciones esenciales del saber en cuestión, así como por el reconocimiento de los temas que lo contextualizan, por la posibilidad de reconocer sus diversas perspectivas y por la capacidad de transferir los principios del saber a otros campos lejanos (Mena, 2000).
Los principios del aprendizaje que permite crear, es decir, las relaciones y tensiones esenciales, las relaciones cercanas, las multiperspectivas y la transferencia a campos lejanos (Mena, 2000), se pueden considerar cartas de navegación para la metodología en cuestión. Ésta propone una planificación de la enseñanza en la que se distinguen fases;

- unas en las que el trabajo tiene que ver con la identificación de temas esenciales, relaciones y perspectivas relevantes, seleccionando lo más relevante y significativo y

- otras, donde el trabajo es el diseño de actividades que permitan, además de transferir estos contenidos, estimular las habilidades para la creatividad.

De esta manera, la creatividad se estimula a través de varios tipos de actividades, que van acostumbrando al estudiante a una epistemología y a una actitud frente al conocimiento y la vida.

Esta propuesta se apoya en marcos teóricos de diversa índole. El marco teórico de la creatividad, es una construcción que mezcla varias fuentes y epistemologías. Una, es la vertiente humanista, especialmente en lo referido a la relación que estimula la actitud creativa y los aspectos afectivos del potencial creador (Rogers, 1976). Otra vertiente la constituyen las propuestas derivadas de estudios descriptivos norteamericanos respecto de las características de las personas creativas. Estas propuestas, unidas a los estudios de investigadores y teóricos del pensamiento creativo como Torrance (1976), Osborn (1953) y Guildford (1980), aportan acerca de los factores cognitivos factibles de estimular/educar.

Un segundo antecedente teórico que apoya la investigación, es el que brinda la vertiente crítica, fundamentalmente a través de Bernstein (1971, 1973). Se trata de buscar marcos epistemológicos para la enseñanza 
que permitan lo que el autor llama "códigos elaborados"; esto es, en la transmisión de saberes profundos, a nivel conceptual, en un proceso que permita la apropiación y resignificación de lo aprendido. Freire también aporta a este marco con material referido a la búsqueda de relaciones y lenguajes que permiten transferir códigos que faciliten a los alumnos la adquisición de aprendizajes que posibiliten transformar sus contextos y movilizarse socialmente.

Complementariamente a la vertiente crítica, el tercer atecedente teórico es el brindado por la filosofía del lenguaje. Wittgenstein (1988) concibe el lenguaje como un juego normado por reglas que permiten múltiples usos (y abusos); Austin (1971, 1990) y Searle $(1977,1990)$ investigan en este mismo sentido lo que el hablante (en este caso el profesor) hace cuando habla. Desde esta perspectiva, se ha planteado como hipótesis de trabajo que habría ciertos actos de habla que favorecerían la participación y el pensamiento de más alto orden.

Un estudio cuasi experimental con grupo de control, en seis liceos con contextos y rendimientos de nivel promedio o alto, mostró un efecto significativo del método, tanto en la adquisición de conocimientos declarativos como procedurales, sugiriendo una relación entre enseñanza para la creatividad y aprendizaje profundo.

\section{Análisis}

\section{Contextos que hacen posible una escuela donde se aprenda para crear.}

Si la creatividad se asocia con aprendizaje profundo, tal como lo sugiere la última investigación citada, entonces se transforma en un imperativo volver a la exploración de los contextos que hacen posible esta enseñanza. Es aquí donde, luego de haber recorrido las investigaciones previas que sustentan el propósito de este artículo, llegamos al foco de discusión: los contextos que permiten enseñar para la creatividad y para el aprendizaje, ambos objetivos de la sociedad del conocimiento y el cambio.

El análisis se realizará relacionando los principios de las cuatro investigaciones citadas:

1.- Los principios para la gestión de contextos educativos que liberen la creatividad, derivados del estudio de demandas sociales de creatividad, de Mena (1995).

2.- El análisis de la investigación referida a un intervención global para mejorar el contexto (Fondecyt 1980610, 1999-2002), que mostró el efecto negativo de ciertos contextos escolares sobre estrategias para la creatividad.

3.- El citado estudio correlaciones de Dyer y Meyer (2000) acerca de las características de grupos creativos.

4.- Lo que se deriva de las propuestas recogidas en focus groups de los profesores que trabajaron exitosamente con el método de Aprender para Crear (Fondecyt 1010981, 2002-2004).

\section{Dos principios para una gestión liberadora:}

\section{Organización descentralizada en función} del aprendizaje significativo de todos los estudiantes.

Pareciera necesario concluir la descentralización iniciada en 1981 con la municipalización, y comenzar a implementar una estructura que permita una mejor acción autónoma y responsable del liceo, apoyada por la coordinación y colaboración municipal o de la entidad sostenedora pertinente. Esto requiere estar profundamente ligado a una aclaración del objeto de la educación y a la racionalidad de dicho objeto: el aprendizaje de calidad para todos. No puede separarse el 
tema de la gestión del tema del objeto de la educación, y tampoco asumirse que el objeto esté claro. Educar a todos no significa sólo cumplir una meta del paradigma educativo tradicional, sino que requiere un cambio de paradigma educativo. Este nuevo paradigma, que pone el foco en las diferencias individuales, en los contextos familiares y comunitarios, y en los proyectos de vida, induce otro paradigma de gestión, que podemos llamar "gestión centrada en el apoyo al aula".

Algunas luces y medidas estratégicas para el tránsito descentralizador de la responsabilidad frente a un objetivo relevante podrían ser:

\section{Calidad y sentido claro: objeto de la creatividad}

- Propuestas explicadas en sus bases más profundas.

- El trabajo del aula en el centro.

- Foco en la igualdad de resultados (justicia y solidaridad), no de la oferta.

\section{Credibilidad y confianza: posibilidad de crear}

- El clima positivo como garante de credibilidad.

- No eludir significados ni la emocionalidad de los docentes.

- Predecir y aclarar posiciones post cambios.

- Permanencia en el cargo ligada a resultados.

- Estructura burocrática mínima, en función de objetivos relevantes.

- Información.

- Feedback.

- Evaluar variables sobre las que se tiene control.
- Evaluar impacto sobre el aprendizajes.

- Procesos orgánicos de evaluación/ incentivo.

- Evaluaciones: generativas.

- Sistemas de corrección durante el proceso.

- Tutorías, feedback individuales, grupos focales.

- Establecer fórmulas de seguimiento.

- Exigencias altas.

- Flexibilizar demandas de resultados en función de las necesidades y problemas.

\section{Identidad, libertad y posibilidad de} cometer errores en función de la búsqueda de calidad educativa.

El segundo principio del contexto que permite la creatividad, se refiere a la claridad que la comunidad docente y directiva de una institución debe tener respecto del tipo de comportamiento humano que le da sentido y razón de ser a una escuela -aprender y enseñar-. Esta claridad, junto a una verdadera comprensión de las bases motivacionales del aprendizaje y de la enseñanza, debe estar a la base de la organización de todo el quehacer, para que esté en función de lograr la motivación requerida para el comportamiento deseado.

Educar y aprender conocimientos escolares no tiene un impacto inmediato sobre la vida de las personas. Según Maslow estos conocimientos tendrían un nivel superior en la jerarquía de necesidades, lo que significa que la ausencia de buenas prácticas no amenaza la sobrevivencia inmediata de nadie. De lo anterior se desprende que si aprender exige un esfuerzo, y si no hay motivación para 
hacerlo, la probabilidad de que se haga es nula. Esto implica en primer lugar, la necesidad de entender las bases motivacionales del aprendizaje, para así lograr que los estudiantes tengan interés por aprender $\mathrm{y}$, con ello, lograr que la actividad de enseñar tenga alguna probabilidad de suceder y de tener un impacto significativo. Este interés es uno que va más allá de cumplir con la obligación que le dará la certificación de enseñanza media, que permita encontrar trabajo o entrar a la universidad. En el caso de los docentes, se trata de encontrar las bases motivacionales para enseñar, más allá de contar con un trabajo que les dé sustento.

Hay tres principios que coinciden con el contexto que motiva para aprender y crear:

- Un contexto que respeta a todos por igual, permitiendo y estimulando la identidad de cada uno: Respeta a las personas en su calidad de iguales y en su diferencia individual, estimulando la autenticidad en los pensamientos, palabras y obras. Esto es contrario a la idea de verdades universales, hegemonía cultural, sectarismos de clase, edad y raza.

- Un contexto que otorga libertades para buscar el propio camino: Ofrece alternativas que son susceptibles de ser modificadas, en cuanto a estrategias y programas, de acuerdo a las propias características de la comunidad. Un contexto democrático en la toma de decisiones.

- Un contexto que, pese a la exigencia, permite el error (producto de nuestra búsqueda).

\section{Discusión}

La dificultad de contar con voluntades políticas para desarrollar contextos que estimulen la creatividad en las escuelas, se encuentra con que las sociedades necesitan ciudadanos creativos, y con que los estudiantes ven un sin sentido en los contextos esco- lares. Pero este es un encuentro sin conflicto y justamente ese es el problema. Los alumnos no cuestionan a la escuela en sus bases ontológicas ni en sus propuestas programáticas. Como si los alumnos, que son quienes podrían poner el dedo en la llaga, creyeran que su descontento no tiene bases valederas, como si no captaran que lo que los aflige tiene un componente que atenta directamente contra ellos. Pero sí lo hace, porque este contexto bloquea la creatividad de todo ser humano. La sociedad por su parte, no cree que el contexto relacional sea responsable de los problemas de rendimiento, sino más bien pone el énfasis en la capacidad de los profesores para enseñar. El contexto pasa al plano de "telón del fondo", guardando el secreto del descontento y de la ineficacia. Es lo habitual en la historia del sistema educativo, que repite el rol de reproducción.

Sin embargo, la lucha por la dignidad del sistema educativo obliga a seguir llamando la atención sobre el contexto de relaciones, así como del sistema de representaciones y acuerdos que hace del contexto escolar de nuestros países un lugar donde casi nadie está realmente satisfecho, donde nadie siente que la educación que recibe le permite desarrollar su propio proyecto de vida. Las investigaciones realizadas en estos años nos muestran que es posible hacer las cosas de otra manera, y que cuando se sigue este otro camino las personas recobran su emocionalidad y el sentido existencial, dándose cuenta de que hay más "saberes" en las personas de los que comúnmente activan, y que es el contexto el que bloquea el potencial existente. Los resultados, el análisis y las discusiones también entregan pistas valiosas para el cambio de estos contextos, pistas claras y sencillas, pero que sin duda obedecen a otro paradigma: un paradigma cuya base está en que confía en la potencia creadora de las personas cuando se les permite más libertad, cuando no se espera tenerlo todo controlado y cuando se toma en serio el trabajo, como un asunto en el que la vida está comprome- 
tida. Los profesores pueden morir existencialmente enseñando con modos en los que no creen, materias que no han sopesado en su valor; y los alumnos pueden morir doce años de sus vidas aprendiendo superficialidades, ejercitando músculos periféricos. Así también, está la posibilidad, que algunos toman, de tomarse la enseñanza y el aprendizaje en serio, y trabajar trascendiendo lo habitual, lo establecido, "lo que se pide".

Un buen incentivo para hacerlo es saber que los colegios que han tomado esta última opción, no sólo no han tenido represalias de parte de los organismos administrativos y estatales, sino por el contrario, han logrado aplausos por sus éxitos, tanto en rendimiento como en satisfacción de apoderados, alumnos y profesores. Lo demuestran las investigaciones acerca de los colegios exitosos que ha hecho la UNICEF en los últimos años, las del Ministerio de Educación. Eso sí, cambiar el contexto hacia uno que favorezca la creatividad tiene el costo de despertarse, salir de la modorra y darse una ducha: cosa que siempre cuesta un poco.

\section{Bibliografía}

Antonijevic, N., Mena, I. (1989). El concepto de creatividad. En Creatividad: desafío al sistema educacional. Santiago: CPU.

Austin, J. (1990). Cómo hacer cosas con palabras, Barcelona: Paidós.

Bernstein, B. (1971). Class, codes and control. Londres: Routledge and Kegan Paul.

Bernstein, B. (1973). Education cannot compensate for society. En D. Rubinstein \& C. Stoneman. (Eds). Education for democracy. Londres: Penguin.

Castro, E. (1989). Bases culturales de la creatividad. En Creatividad: desafío al sistema educacional. Santiago: CPU.
Electorat, L., Machuca, A., \& Rauld, X. (1991). Hacia un concepto de creatividad culturalmente pertinente. Tesis para optar al Título de Psicólogo. Santiago: Pontificia Universidad Católica de Chile.

Hirsch, J. (1996). The schools we need and why we don't have them. Revista de Estudios Públicos, Nr. 66, Santiago: CEP.

Fullan, M., Stigelbauer, S. (1997). El Cambio Educativo. México: Trillas.

GuILDFORD, J.P. (1980). La Creatividad. En Beudot (1980), La Creatividad. España: Narcea.

López, R. (1999) Prontuario de la Creatividad. Santiago: Universidad Educares.

MenA, I. (1994). Reflexiones imprescindibles para la incorporación de la creatividad en el sistema educacional chileno. En López e I. Mena (Eds.) Las ovejas y el infinito: propuestas para el desarrollo de la creatividad en el sistema educacional. Santiago: CPU.

Mena, I. (1992). El oculto currículum de la creatividad. Santiago: CPU.

Mena, I., Vizcarra, R. \& Catalán, J. (2000). Estudio de impacto de una estrategia para el desarrollo de la creatividad. Santiago: Fondecyt 1980610, 1998-2000

Mena, I., Rittershausen, S. y Sepúlveda, J. (1994). Educación media y perfeccionamiento docente: la visión de los profesores. Chile: CPU.

Mena, I. (1995). Creatividad con reflexión ética: un estudio desde las demandas sociales. Tesis para optar al Título de Doctorado en Ciencias de la Educación. Santiago: Pontificia Universidad Católica de Chile.

Mena, I. (2000). Aprender para crear. Revista Psykhe. Santiago de Chile: Escuela de Psicología. Pontificia Universidad Católica de Chile. 
OteIZA, F. (1994). Currículum y enseñanza media. Chile: MINEDUC.

Pérez Gómez (1985). Conocimiento académico y aprendizaje significativo. En Pérez Gómez \& G. Sacristán. "La enseñanza su teoría y su práctica". España: Akal editor.

Pérez Gómez. (1985). Paradigmas contemporáneos de investigación didáctica. En Pérez Gómez \& G. Sacristán. La enseñanza su teoría y su práctica. España: Akal editor.

Rogers, C. (1976). Libertad y creatividad en Educación. Buenos Aires: Paidos.

SACristán, G. (1985). Planificación de la investigación educacional y su impacto en la realidad". En Pérez Gómez \& G. Sacristán. La enseñanza su teoría y su práctica. España: Akal editor.

Sepúlveda, G. (2001). Teoría de la actividad. Santiago: Fondecyt 1010931.

VizCArra, R. (1996). "Participación en el aula: una mirada desde los actos de habla". Tesis para optar al Título de Doctorado en Ciencias de la Educación. Santiago: Pontificia Universidad Católica de Chile

\section{Bibliografía de Referencia:}

SANTOS, M. (coordinador) (2003). Aprender a convivir en la escuela. Madrid: Universidad Internacional de Andalucía, Akal.

Garrido, J. (2004) \z «http:// 146.155.39.20:8991/exlibris / aleph / u16_1/alephe/www_f_spa/icon / fseparator.gif»Caracterización de la racionalidad presente en los actores que participan en los procesos de construcción de planes y programas propios para el primer año de la enseñanza humanístico científica. Santiago: Pontificia Universidad Católica. Tesis (Magíster en Ciencias de la Educación)
Redorta, J (2004) Cómo analizar los conflictos: la tipología de conflictos como herramienta de mediación. Barcelona: Paidós

Calcagni, A. (2003) Aprendizaje y emocionalidad. Notas Preliminares. En Revista Pensamiento Educativo, Vol. 28. Santiago

IbÁÑeZ, N., Barrientos, F., Delgado, T., FigueroA, A., Geisse, G. (2004) Las emociones en el aula y la calidad de la educación. En Revista Pensamiento Educativo, Vol. 35. Santiago.

Montanero, M., García, G. (2005) What do teachers do when students make a mistake? An analysis of verbal interaction in an educational support classroom. En revista Infancia y Aprendizaje. Vol.28, N². España: Fundación Infancia y Aprendizaje.

Garaigordobil, M. (2005) Assessment of changes in creative personality behaviours and traits: Effects of a programme for children aged 10-11 years. En revista Infancia y Aprendizaje, Vol.28, N 1 . España: Fundación Infancia y Aprendizaje.

Horng, Jeou-Shyan; Hong, Jon-ChaO; ChanLin, LiH-Juan; Chang, ShiH-Hui; Chu, Hui-Chuan (2005) Creative teachers and creative teaching strategies. En International Journal of Consumer Studies, Vol. 29, Number 4. USA: Blackwell Publishing

Fecha Recepción Artículo: 1 de marzo 2005 Fecha Evaluación Final: 22 de mayo 2006 